\title{
3D Ising system in an external field. Recurrence relations for the asymmetric $\rho^{6}$ model
}

\author{
I.V.Pylyuk, M.P.Kozlovskii \\ Institute for Condensed Matter Physics \\ of the National Academy of Sciences of Ukraine, \\ 1 Svientsitskii Str., 79011 Lviv, Ukraine
}

Received July 26, 2000

\begin{abstract}
The 3D one-component spin system in an external magnetic field is studied using the collective variables method. The integration of the partition function of the system over the phase space layers is performed in the approximation of the sextic measure density including the even and the odd powers of the variable (the asymmetric $\rho^{6}$ model). The general recurrence relations between the coefficients of the effective measure densities are obtained. The new functions appearing in these recurrence relations are given in the form of a convergent series.
\end{abstract}

Key words: Ising model, external field, collective variables, recurrence relations

PACS: $05.50 .+q, 75.10 . H k$

\section{Introduction}

The object of investigation is the Ising model on a simple cubic lattice with an exponentially decreasing interaction potential. The Ising model, which is simple and convenient for mathematical analysis, is widely used in the theory of phase transitions for an analysis of properties of various magnetic and nonmagnetic systems (binary mixtures, lattice model of liquids, ferromagnets, etc.).

The three-dimensional (3D) Ising system in an external magnetic field will be studied here based on the collective variables (CV) method [1]. This original method can be extended to the liquid-gas system [2], multicomponent fluids [3]. In this case, the method makes it possible to use a convergent series by applying the solution of the 3D Ising model in contrast to the asymptotic $\epsilon$-expansions (see, for example, $[4,5])$.

The CV method allows one to calculate the partition function of the Ising model and to obtain not only the universal quantities but also analytic expressions for 
nonuniversal characteristics as functions of the microscopic parameters of the system. The statistical description of the spin system behaviour is performed in real 3D space on the microscopic level. The main results obtained in the CV method for the 3D systems in the absence of an external field are presented in $[1,6]$.

An important condition for describing the system properties by the CV method is the use of non-Gaussian measure densities [1]. Such a measure density at a zero external field is represented as an exponential function of the CV, the argument of which contains, along with the quadratic term, higher even powers of the variable with the corresponding coupling constants. The confinement to the 6 th power of the $\mathrm{CV}$ in the expression for a measure density corresponds to the $\rho^{6}$ model. This model provides a more adequate quantitative description of the 3D Ising system behaviour in comparison with the $\rho^{4}$ model (see [7-9]).

In the case of a nonzero external field, the non-Gaussian measure density includes the terms proportional to odd powers of the $\mathrm{CV}$ in addition to the terms proportional to even powers. The partition function of the $3 \mathrm{D}$ one-component system in an external field is presented below in the approximation of the sextic measure density, which involves the even and odd powers up to the 6th power of the variable (the asymmetric $\rho^{6}$ model). A partition function functional is similar to the GinzburgLandau functional (see, for example, [10]). We calculate the partition function by successively integrating its expression over the short-wave or the so-called unimportant variables. The corresponding renormalization group ( $\mathrm{RG}$ ) transformation can be related to the Wilson type [11]. The general recurrence relations (RR) corresponding to the asymmetric $\rho^{6}$ model and the new special functions entering the $\mathrm{RR}$ are considered.

In our earlier works (see, for example, [7-9]), an infinitely weak external field was introduced in the course of calculation of the contribution from the long-wave modes of the spin moment density oscillations to the 3D Ising system thermodynamic characteristics. In this paper, we introduce an external field in the Hamiltonian from the outset. Such an approach leads to the appearance of odd powers of the CV in the expression for the partition function and makes it possible to describe a lot of quantities (cumulants in the Jacobian of the transition to the CV set, initial coefficients of the partition function, etc.) as functions of an external field.

\section{Recurrence relations for the asymmetric $\rho^{6}$ model}

We consider the Ising system on a simple cubic lattice with the period $c$. The Hamiltonian of the system has the form

$$
H=-\frac{1}{2} \sum_{\mathbf{i}, \mathbf{j}} \Phi\left(r_{\mathbf{i j}}\right) \sigma_{\mathbf{i}} \sigma_{\mathbf{j}}-h \sum_{\mathbf{i}} \sigma_{\mathbf{i}}
$$

Here $h$ is an external field, $r_{\mathbf{i j}}$ is the distance between particles at the sites $\mathbf{i}$ and $\mathbf{j}, \sigma_{\mathbf{i}}$ is the operator of the $z$-component of the spin at the ith site, having two eigenvalues 
+1 and -1 . The interaction potential is an exponentially decreasing function

$$
\Phi\left(r_{\mathbf{i j}}\right)=A \exp \left(-\frac{r_{\mathbf{i j}}}{b}\right)
$$

where $A$ is a constant, $b$ is the radius of effective interaction. For the Fourier transform of the interaction potential, we use the following approximation:

$$
\tilde{\Phi}(k)= \begin{cases}\tilde{\Phi}(0)\left(1-2 b^{2} k^{2}\right), & k \leqslant B^{\prime}, \\ 0, & B^{\prime}<k \leqslant B .\end{cases}
$$

Here $B=\pi / c$ is the boundary of the Brillouin half-zone, $B^{\prime}=(b \sqrt{2})^{-1}$ is determined from the condition for the application of the parabolic approximation $\tilde{\Phi}(0)\left(1-2 b^{2} k^{2}\right)=0, \tilde{\Phi}(0)=8 \pi A(b / c)^{3}$. At $\tilde{\Phi}(0)=2 d J, b=b_{I}=c /(2 \sqrt{d})(J$ is the constant of the interaction between the nearest neighbours, $d=3$ is the space dimension) for small values of the wave vectors $\mathbf{k}$, the parabolic approximation of the Fourier transform of the exponentially decreasing interaction potential corresponds to the analogous approximation of the Fourier transform for the interaction potential between the nearest neighbours [12].

In the CV representation for the partition function of the 3D Ising model in an external field, we have

$$
Z=\int \exp \left[\frac{1}{2} \sum_{\mathbf{k}} \beta \tilde{\Phi}(k) \rho_{\mathbf{k}} \rho_{-\mathbf{k}}\right] J(\rho)(\mathrm{d} \rho)^{N},
$$

where the summation over the wave vectors $\mathbf{k}$ is carried out within the first Brillouin zone, $\beta=1 /\left(k_{\mathrm{B}} T\right)$ is the inverse thermodynamic temperature, $k_{\mathrm{B}}$ is the Boltzmann constant. The $\mathrm{CV} \rho_{\mathbf{k}}$ are introduced using the relations of the type of an analytic functional for operators of spin density oscillation modes $\hat{\rho}_{\mathbf{k}}=(\sqrt{N})^{-1} \sum_{\mathbf{l}} \sigma_{\mathbf{l}}$. $\exp (-\mathrm{ikl})[1]$. The Jacobian of the transition from the set of the $N$ spin variables $\sigma_{\mathbf{l}}$ to the set of the $\mathrm{CV} \rho_{\mathbf{k}}$ can be written as

$$
\begin{aligned}
J(\rho)= & \left(2 \cosh h^{\prime}\right)^{N} \int \exp \left[2 \pi \mathrm{i} \sum_{\mathbf{k}} \omega_{\mathbf{k}} \rho_{\mathbf{k}}\right. \\
& \left.+\sum_{n \geqslant 1}(-2 \pi \mathrm{i})^{n} N^{-(n-2) / 2} \frac{\mathcal{M}_{n}}{n !} \sum_{\mathbf{k}_{1}, \ldots, \mathbf{k}_{n}} \omega_{\mathbf{k}_{1}} \cdots \omega_{\mathbf{k}_{n}} \delta_{\mathbf{k}_{1}+\cdots+\mathbf{k}_{n}}\right](\mathrm{d} \omega)^{N} .
\end{aligned}
$$

Here $\delta_{\mathbf{k}_{1}+\cdots+\mathbf{k}_{n}}$ is the Kronecker symbol, the variables $\omega_{\mathbf{k}}$ are conjugate to $\rho_{\mathbf{k}}$. The cumulants $\mathcal{M}_{n}$ are functions of a generalized field $h^{\prime}=\beta h$ :

$$
\begin{aligned}
& \mathcal{M}_{1}=\tanh h^{\prime}=x, \\
& \mathcal{M}_{2}=1-\tanh ^{2} h^{\prime}=y, \\
& \mathcal{M}_{3}=-2 x y \\
& \mathcal{M}_{4}=-2 y^{2}+4 x^{2} y, \\
& \mathcal{M}_{5}=16 x y^{2}-8 x^{3} y \\
& \mathcal{M}_{6}=16 y^{3}-88 x^{2} y^{2}+16 x^{4} y .
\end{aligned}
$$


We shall proceed from the expression for the partition function in the approximation of the asymmetric $\rho^{6}$ model. Putting $n=6$ in (2.5) and carrying out integration in (2.4) with respect to the variables $\rho_{\mathbf{k}}$ and $\omega_{\mathbf{k}}$ with indices $B^{\prime}<k \leqslant B$, followed by the integration with respect to the $N^{\prime}$ variables $\omega_{\mathbf{k}}$, we obtain

$$
\begin{aligned}
Z= & \left(2 \cosh h^{\prime}\right)^{N} 2^{\left(N^{\prime}-1\right) / 2} \mathrm{e}^{a_{0}^{\prime} N^{\prime}} \int \exp \left[-\left(N^{\prime}\right)^{1 / 2} a_{1}^{\prime} \rho_{0}-\frac{1}{2} \sum_{k \leqslant B^{\prime}} d^{\prime}(k) \rho_{\mathbf{k}} \rho_{-\mathbf{k}}\right. \\
& \left.-\sum_{l=3}^{6} \frac{a_{l}^{\prime}}{l !\left(N^{\prime}\right)^{l / 2-1}} \sum_{k_{1}, \ldots, k_{l} \leqslant B^{\prime}} \rho_{\mathbf{k}_{1}} \cdots \rho_{\mathbf{k}_{l}} \delta_{\mathbf{k}_{1}+\cdots+\mathbf{k}_{l}}\right](\mathrm{d} \rho)^{N^{\prime}},
\end{aligned}
$$

where $N^{\prime}=N s_{0}^{-d}, s_{0}=B / B^{\prime}=\pi \sqrt{2} b / c$, and

$$
d^{\prime}(k)=a_{2}^{\prime}-\beta \tilde{\Phi}(k) \text {. }
$$

The coefficients

$$
\begin{aligned}
& a_{0}^{\prime}=\ln Q(\mathcal{M}), \quad Q(\mathcal{M})=\pi^{-1}\left(\frac{4 !}{\overline{\mathcal{M}}_{4}} s_{0}^{d}\right)^{1 / 4} I_{0 c}\left(\eta^{\prime}\right), \\
& a_{1}^{\prime}=-\left(\frac{4 !}{\overline{\mathcal{M}}_{4}} s_{0}^{d}\right)^{1 / 4} C_{1 s c}\left(\eta^{\prime}\right), \\
& a_{k}^{\prime}=\left(\frac{4 !}{\overline{\mathcal{M}}_{4}} s_{0}^{d}\right)^{k / 4} C_{k s c}\left(\eta^{\prime}\right), \quad k=2,3,4,5,6
\end{aligned}
$$

are functions of a field $h^{\prime}$ and a ratio $b / c$. In this expressions, $\overline{\mathcal{M}}_{4}=-\mathcal{M}_{4}$. The set $\eta^{\prime}=\left\{\eta_{1}^{\prime}, \eta_{2}^{\prime}, \eta_{3}^{\prime}, \eta_{4}^{\prime}, \eta_{5}^{\prime}, \eta_{6}^{\prime}\right\}$ is the set of initial arguments

$$
\begin{array}{rlrl}
\eta_{1}^{\prime} & =\mathcal{M}_{1}\left(\frac{4 !}{\overline{\mathcal{M}}_{4}}\right)^{1 / 4} s_{0}^{3 d / 4}, & \eta_{2}^{\prime}=\frac{\mathcal{M}_{2}}{2}\left(\frac{4 !}{\overline{\mathcal{M}}_{4}}\right)^{1 / 2} s_{0}^{d / 2} \\
\eta_{3}^{\prime}=\frac{\mathcal{M}_{3}}{3 !}\left(\frac{4 !}{\overline{\mathcal{M}}_{4}}\right)^{3 / 4} s_{0}^{d / 4}, & \eta_{4}^{\prime} \equiv 1, \\
\eta_{5}^{\prime}=\frac{\mathcal{M}_{5}}{5 !}\left(\frac{4 !}{\overline{\mathcal{M}}_{4}}\right)^{5 / 4} s_{0}^{-d / 4}, & \eta_{6}^{\prime}=\frac{\mathcal{M}_{6}}{6 !}\left(\frac{4 !}{\overline{\mathcal{M}}_{4}}\right)^{3 / 2} s_{0}^{-d / 2} .
\end{array}
$$

The special functions $C_{l s c}\left(\eta^{\prime}\right)$ in (2.9) are combinations of the functions $F_{(2 l+1) s c}\left(\eta^{\prime}\right)=I_{(2 l+1) s}\left(\eta^{\prime}\right) / I_{0 c}\left(\eta^{\prime}\right)$ and $F_{2 l c c}\left(\eta^{\prime}\right)=I_{2 l c}\left(\eta^{\prime}\right) / I_{0 c}\left(\eta^{\prime}\right)$ :

$$
\begin{aligned}
C_{1 s c}\left(\eta^{\prime}\right)= & F_{1 s c}\left(\eta^{\prime}\right), \\
C_{2 s c}\left(\eta^{\prime}\right)= & F_{2 c c}\left(\eta^{\prime}\right)+F_{1 s c}^{2}\left(\eta^{\prime}\right), \\
C_{3 s c}\left(\eta^{\prime}\right)= & F_{3 s c}\left(\eta^{\prime}\right)-3 F_{2 c c}\left(\eta^{\prime}\right) F_{1 s c}\left(\eta^{\prime}\right)-2 F_{1 s c}^{3}\left(\eta^{\prime}\right), \\
C_{4 s c}\left(\eta^{\prime}\right)= & -F_{4 c c}\left(\eta^{\prime}\right)-4 F_{3 s c}\left(\eta^{\prime}\right) F_{1 s c}\left(\eta^{\prime}\right)+3 F_{2 c c}^{2}\left(\eta^{\prime}\right)+12 F_{2 c c}\left(\eta^{\prime}\right) F_{1 s c}^{2}\left(\eta^{\prime}\right) \\
& +6 F_{1 s c}^{4}\left(\eta^{\prime}\right), \\
C_{5 s c}\left(\eta^{\prime}\right)= & -F_{5 s c}\left(\eta^{\prime}\right)+5 F_{4 c c}\left(\eta^{\prime}\right) F_{1 s c}\left(\eta^{\prime}\right)+10 F_{3 s c}\left(\eta^{\prime}\right) F_{2 c c}\left(\eta^{\prime}\right)
\end{aligned}
$$




$$
\begin{aligned}
& +20 F_{3 s c}\left(\eta^{\prime}\right) F_{1 s c}^{2}\left(\eta^{\prime}\right)-30 F_{2 c c}^{2}\left(\eta^{\prime}\right) F_{1 s c}\left(\eta^{\prime}\right)-60 F_{2 c c}\left(\eta^{\prime}\right) F_{1 s c}^{3}\left(\eta^{\prime}\right) \\
& -24 F_{1 s c}^{5}\left(\eta^{\prime}\right) \\
C_{6 s c}\left(\eta^{\prime}\right)= & F_{6 c c}\left(\eta^{\prime}\right)+6 F_{5 s c}\left(\eta^{\prime}\right) F_{1 s c}\left(\eta^{\prime}\right)-15 F_{4 c c}\left(\eta^{\prime}\right) F_{2 c c}\left(\eta^{\prime}\right) \\
& -30 F_{4 c c}\left(\eta^{\prime}\right) F_{1 s c}^{2}\left(\eta^{\prime}\right)+10 F_{3 s c}^{2}\left(\eta^{\prime}\right)-120 F_{3 s c}\left(\eta^{\prime}\right) F_{2 c c}\left(\eta^{\prime}\right) F_{1 s c}\left(\eta^{\prime}\right) \\
& -120 F_{3 s c}\left(\eta^{\prime}\right) F_{1 s c}^{3}\left(\eta^{\prime}\right)+30 F_{2 c c}^{3}\left(\eta^{\prime}\right)+270 F_{2 c c}^{2}\left(\eta^{\prime}\right) F_{1 s c}^{2}\left(\eta^{\prime}\right) \\
& +360 F_{2 c c}\left(\eta^{\prime}\right) F_{1 s c}^{4}\left(\eta^{\prime}\right)+120 F_{1 s c}^{6}\left(\eta^{\prime}\right)
\end{aligned}
$$

Here

$$
I_{(2 l+1) s}\left(\eta^{\prime}\right)=\int_{0}^{\infty} t^{2 l+1} f_{s}(t) \mathrm{d} t, \quad I_{2 l c}\left(\eta^{\prime}\right)=\int_{0}^{\infty} t^{2 l} f_{c}(t) \mathrm{d} t, \quad l=0,1,2,3,
$$

and

$$
\begin{aligned}
& f_{s}(t)=\sin \left(\eta_{1}^{\prime} t-\eta_{3}^{\prime} t^{3}+\eta_{5}^{\prime} t^{5}\right) \exp \left(-\eta_{2}^{\prime} t^{2}-\eta_{4}^{\prime} t^{4}-\eta_{6}^{\prime} t^{6}\right), \\
& f_{c}(t)=\cos \left(\eta_{1}^{\prime} t-\eta_{3}^{\prime} t^{3}+\eta_{5}^{\prime} t^{5}\right) \exp \left(-\eta_{2}^{\prime} t^{2}-\eta_{4}^{\prime} t^{4}-\eta_{6}^{\prime} t^{6}\right) .
\end{aligned}
$$

We use the method of layer-by-layer integration of (2.7) with respect to the variables $\rho_{\mathbf{k}}$ proposed by I.R.Yukhnovskii [1]. The integration begins from the variables $\rho_{\mathbf{k}}$ with large values of $k$ (of the order of the Brillouin half-zone boundary). The phase space of the $\mathrm{CV} \rho_{\mathbf{k}}$ is divided into layers with the division parameter $s$. In each $n$th layer (corresponding to the region of wave vectors $B_{n+1}<k \leqslant B_{n}$, $\left.B_{n+1}=B_{n} / s, s>1\right)$, the Fourier transform of the potential $\tilde{\Phi}(k)$ is replaced by its average value $\tilde{\Phi}\left(B_{n+1}, B_{n}\right)$. As a result of step-by-step calculation of the partition function, the number of integration variables in the expression for this quantity decreases gradually. The partition function is then represented as a product of the partial partition functions of individual layers and the integral of the "smoothed" effective measure density. After the integration over the $n+1$ layers of the CV space, we obtain

$$
Z=\left(2 \cosh h^{\prime}\right)^{N} 2^{\left(N_{n+1}-1\right) / 2} Q_{0} Q_{1} \cdots Q_{n}\left[Q\left(P_{n}\right)\right]^{N_{n+1}} \int \mathcal{W}_{6}^{(n+1)}(\rho)(\mathrm{d} \rho)^{N_{n+1}},
$$

where $N_{n+1}=N^{\prime} s^{-d(n+1)}$, and

$$
\begin{aligned}
& Q_{0}=\left[\mathrm{e}^{a_{0}^{\prime}} Q(d)\right]^{N^{\prime}}, \quad Q_{1}=\left[Q(P) Q\left(d_{1}\right)\right]^{N_{1}}, \quad \ldots, \quad Q_{n}=\left[Q\left(P_{n-1}\right) Q\left(d_{n}\right)\right]^{N_{n}}, \\
& Q\left(d_{n}\right)=\left(4 ! / a_{4}^{(n)}\right)^{1 / 4} I_{0}\left(h^{(n)}\right), \\
& Q\left(P_{n}\right)=\pi^{-1}\left(s^{d} a_{4}^{(n)} / C_{4}\left(h^{(n)}\right)\right)^{1 / 4} I_{0 c}\left(\eta^{(n)}\right) .
\end{aligned}
$$

The components

$$
h_{1}^{(n)}=a_{1}^{(n)}\left(\frac{4 !}{a_{4}^{(n)}}\right)^{1 / 4}, \quad h_{2}^{(n)}=\frac{d_{n}\left(B_{n+1}, B_{n}\right)}{2}\left(\frac{4 !}{a_{4}^{(n)}}\right)^{1 / 2},
$$




$$
\begin{array}{ll}
h_{3}^{(n)}=\frac{a_{3}^{(n)}}{3 !}\left(\frac{4 !}{a_{4}^{(n)}}\right)^{3 / 4}, & h_{4}^{(n)} \equiv 1, \\
h_{5}^{(n)}=\frac{a_{5}^{(n)}}{5 !}\left(\frac{4 !}{a_{4}^{(n)}}\right)^{5 / 4}, & h_{6}^{(n)}=\frac{a_{6}^{(n)}}{6 !}\left(\frac{4 !}{a_{4}^{(n)}}\right)^{3 / 2}
\end{array}
$$

of the set of basic arguments in the $n$th layer $h^{(n)}=\left\{h_{1}^{(n)}, h_{2}^{(n)}, h_{3}^{(n)}, h_{4}^{(n)}, h_{5}^{(n)}, h_{6}^{(n)}\right\}$ are determined by the average value of the coefficient $d_{n}(k)$, i.e., by $d_{n}\left(B_{n+1}, B_{n}\right)=$ $a_{2}^{(n)}-\beta \tilde{\Phi}\left(B_{n+1}, B_{n}\right)$ as well as the quantities $a_{1}^{(n)}, a_{3}^{(n)}, a_{4}^{(n)}, a_{5}^{(n)}$ and $a_{6}^{(n)}$. The components of the set of intermediate arguments $\eta^{(n)}=\left\{\eta_{1}^{(n)}, \eta_{2}^{(n)}, \eta_{3}^{(n)}, \eta_{4}^{(n)}, \eta_{5}^{(n)}, \eta_{6}^{(n)}\right\}$ are functions of the quantities (2.16). They are given by the formulas

$$
\begin{array}{ll}
\eta_{1}^{(n)}=-\left(4 ! s^{3 d}\right)^{1 / 4} C_{1}\left(h^{(n)}\right) C_{4}^{-1 / 4}\left(h^{(n)}\right), & \eta_{2}^{(n)}=\left(6 s^{d}\right)^{1 / 2} C_{2}\left(h^{(n)}\right) C_{4}^{-1 / 2}\left(h^{(n)}\right), \\
\eta_{3}^{(n)}=\left(\frac{32}{3} s^{d}\right)^{1 / 4} C_{3}\left(h^{(n)}\right) C_{4}^{-3 / 4}\left(h^{(n)}\right), & \eta_{4}^{(n)} \equiv 1, \\
\eta_{5}^{(n)}=\frac{\left(4 ! s^{-d}\right)^{1 / 4}}{5} C_{5}\left(h^{(n)}\right) C_{4}^{-5 / 4}\left(h^{(n)}\right), & \eta_{6}^{(n)}=\frac{\left(6 s^{-d}\right)^{1 / 2}}{15} C_{6}\left(h^{(n)}\right) C_{4}^{-3 / 2}\left(h^{(n)}\right) .
\end{array}
$$

The special functions $C_{l}\left(h^{(n)}\right)$ can be expressed in terms of $F_{l}\left(h^{(n)}\right)=$ $I_{l}\left(h^{(n)}\right) / I_{0}\left(h^{(n)}\right)$ :

$$
\begin{aligned}
C_{1}\left(h^{(n)}\right)= & F_{1}\left(h^{(n)}\right), \\
C_{2}\left(h^{(n)}\right)= & F_{2}\left(h^{(n)}\right)-F_{1}^{2}\left(h^{(n)}\right), \\
C_{3}\left(h^{(n)}\right)= & F_{3}\left(h^{(n)}\right)-3 F_{2}\left(h^{(n)}\right) F_{1}\left(h^{(n)}\right)+2 F_{1}^{3}\left(h^{(n)}\right), \\
C_{4}\left(h^{(n)}\right)= & -F_{4}\left(h^{(n)}\right)+4 F_{3}\left(h^{(n)}\right) F_{1}\left(h^{(n)}\right)+3 F_{2}^{2}\left(h^{(n)}\right)-12 F_{2}\left(h^{(n)}\right) F_{1}^{2}\left(h^{(n)}\right) \\
& +6 F_{1}^{4}\left(h^{(n)}\right), \\
C_{5}\left(h^{(n)}\right)= & -F_{5}\left(h^{(n)}\right)+5 F_{4}\left(h^{(n)}\right) F_{1}\left(h^{(n)}\right)+10 F_{3}\left(h^{(n)}\right) F_{2}\left(h^{(n)}\right) \\
& -20 F_{3}\left(h^{(n)}\right) F_{1}^{2}\left(h^{(n)}\right)-30 F_{2}^{2}\left(h^{(n)}\right) F_{1}\left(h^{(n)}\right)+60 F_{2}\left(h^{(n)}\right) F_{1}^{3}\left(h^{(n)}\right) \\
& -24 F_{1}^{5}\left(h^{(n)}\right), \\
C_{6}\left(h^{(n)}\right)= & F_{6}\left(h^{(n)}\right)-6 F_{5}\left(h^{(n)}\right) F_{1}\left(h^{(n)}\right)-15 F_{4}\left(h^{(n)}\right) F_{2}\left(h^{(n)}\right) \\
& +30 F_{4}\left(h^{(n)}\right) F_{1}^{2}\left(h^{(n)}\right)-10 F_{3}^{2}\left(h^{(n)}\right)+120 F_{3}\left(h^{(n)}\right) F_{2}\left(h^{(n)}\right) F_{1}\left(h^{(n)}\right) \\
& -120 F_{3}\left(h^{(n)}\right) F_{1}^{3}\left(h^{(n)}\right)+30 F_{2}^{3}\left(h^{(n)}\right)-270 F_{2}^{2}\left(h^{(n)}\right) F_{1}^{2}\left(h^{(n)}\right) \\
& +360 F_{2}\left(h^{(n)}\right) F_{1}^{4}\left(h^{(n)}\right)-120 F_{1}^{6}\left(h^{(n)}\right) .
\end{aligned}
$$

Here

$$
I_{l}\left(h^{(n)}\right)=\int_{-\infty}^{\infty} t^{l} f^{(n)}(t) \mathrm{d} t, \quad l=0,1,2,3,4,5,6
$$

and

$$
f^{(n)}(t)=\exp \left(-h_{1}^{(n)} t-h_{2}^{(n)} t^{2}-h_{3}^{(n)} t^{3}-h_{4}^{(n)} t^{4}-h_{5}^{(n)} t^{5}-h_{6}^{(n)} t^{6}\right)
$$


The effective sextic measure density of the $(n+1)$ th block structure $\mathcal{W}_{6}^{(n+1)}(\rho)$ is written as

$$
\begin{aligned}
\mathcal{W}_{6}^{(n+1)}(\rho)=\exp [ & -N_{n+1}^{1 / 2} a_{1}^{(n+1)} \rho_{0}-\frac{1}{2} \sum_{k \leq B_{n+1}} d_{n+1}(k) \rho_{\mathbf{k}} \rho_{-\mathbf{k}} \\
& \left.-\sum_{l=3}^{6} \frac{a_{l}^{(n+1)}}{l ! N_{n+1}^{l / 2-1}} \sum_{k_{1}, \ldots, k_{l} \leqslant B_{n+1}} \rho_{\mathbf{k}_{1}} \cdots \rho_{\mathbf{k}_{l}} \delta_{\mathbf{k}_{1}+\cdots+\mathbf{k}_{l}}\right]
\end{aligned}
$$

where $B_{n+1}=B^{\prime} s^{-(n+1)}, d_{n+1}(k)=a_{2}^{(n+1)}-\beta \tilde{\Phi}(k), a_{l}^{(n+1)}$ are the renormalized values of the coefficients $a_{l}^{\prime}$ after the integration over the $n+1$ layers of the phase space of the CV.

The coefficients of the sextic measure densities of the $(n+1)$ th and $n$th block structures are connected through the following general RR:

$$
u_{l}^{(n+1)}=s^{l-\frac{l-2}{2} d}\left[-q \delta_{l-2}+\left(u_{4}^{(n)}\right)^{l / 4} Y_{l}\left(h^{(n)}\right)\right], \quad l=1,2,3,4,5,6 .
$$

We have introduced new designations here:

$$
\begin{aligned}
& u_{1}^{(n)}=s^{n} a_{1}^{(n)}, \\
& u_{2}^{(n)}+q=s^{2 n} d_{n}\left(B_{n+1}, B_{n}\right), \quad u_{2}^{(n)}=s^{2 n} d_{n}(0), \\
& u_{m}^{(n)}=s^{m n} a_{m}^{(n)}, \quad m=3,4,5,6 .
\end{aligned}
$$

The quantity $q=\bar{q} \beta \tilde{\Phi}(0)$ determines the average value of the Fourier transform of the potential in the $n$th layer $\beta \tilde{\Phi}\left(B_{n+1}, B_{n}\right)=\beta \tilde{\Phi}(0)-q / s^{2 n}(\bar{q}$ corresponds to the average value of $k^{2}$ on the interval $\left.(1 / s, 1]\right)$. The functions appearing in the RR (2.22) can be defined by the expressions

$$
\begin{aligned}
& Y_{1}\left(h^{(n)}\right)=s^{-d / 4} C_{1 s c}\left(\eta^{(n)}\right) C_{4}^{-1 / 4}\left(h^{(n)}\right), \\
& Y_{k}\left(h^{(n)}\right)=(-1)^{k} s^{\frac{3 k-4}{4} d} C_{k s c}\left(\eta^{(n)}\right) C_{4}^{-k / 4}\left(h^{(n)}\right), \quad k=2,3,4,5,6 .
\end{aligned}
$$

The functions $C_{l s c}\left(\eta^{(n)}\right)$ have the form similar to $C_{l s c}\left(\eta^{\prime}\right)(2.11)$ under condition that the set $\eta^{\prime}$ and the functions $f_{s}(t), f_{c}(t)(2.13)$ should be replaced by $\eta^{(n)}$ and

$$
\begin{aligned}
& F_{s}(t)=\sin \left(\eta_{1}^{(n)} t+\eta_{3}^{(n)} t^{3}+\eta_{5}^{(n)} t^{5}\right) \exp \left(-\eta_{2}^{(n)} t^{2}-\eta_{4}^{(n)} t^{4}-\eta_{6}^{(n)} t^{6}\right) \\
& F_{c}(t)=\cos \left(\eta_{1}^{(n)} t+\eta_{3}^{(n)} t^{3}+\eta_{5}^{(n)} t^{5}\right) \exp \left(-\eta_{2}^{(n)} t^{2}-\eta_{4}^{(n)} t^{4}-\eta_{6}^{(n)} t^{6}\right)
\end{aligned}
$$

respectively. It should be noted that the formula for $I_{2 l c}\left(\eta^{\prime}\right)$ (see (2.12)) allows us to express the function $I_{0 c}\left(\eta^{(n)}\right)$ entering (2.15) in the same way. The function $C_{4}\left(h^{(n)}\right)$ is given in (2.18).

The obtained expressions make it possible to investigate the properties of the 3D one-component spin system in the vicinity of the critical point $\left(T=T_{c}, h=0\right)$. In the absence of an external field $(h=0)$, we have $a_{1}^{(n)}=a_{3}^{(n)}=a_{5}^{(n)}=0$ and relations 
(2.22) are indentical with the RR presented in [13]. The behaviour of the system can also be studied at $T=T_{\mathrm{c}}$ and $h \rightarrow 0$.

The components of the set of intermediate arguments $\eta^{(n)}$ and the functions $C_{l s c}\left(\eta^{(n)}\right), C_{l}\left(h^{(n)}\right), Y_{l}\left(h^{(n)}\right)$ are approximated by power series in deviations of the basic arguments $h_{i}^{(n)}$ from their values at a fixed point $h_{i}^{*}$. Taking into account the linear deviations, we find the following forms for these series:

$$
\begin{aligned}
A_{l}= & A_{l 0}\left[1+A_{l 1}\left(h_{1}^{*}-h_{1}^{(n)}\right)+A_{l 2}\left(h_{2}^{*}-h_{2}^{(n)}\right)+A_{l 3}\left(h_{3}^{*}-h_{3}^{(n)}\right)\right. \\
& \left.+A_{l 5}\left(h_{5}^{*}-h_{5}^{(n)}\right)+A_{l 6}\left(h_{6}^{*}-h_{6}^{(n)}\right)\right], \quad l=1,2,3,4,5,6 .
\end{aligned}
$$

Here $A_{l}$ are the above-mentioned intermediate arguments or functions. The coefficients $A_{l i}$ are ultimate functions of the basic arguments at a fixed point. It should be emphasized that we necessarily arrive at asymptotic expansions when the Gaussian density of measure is taken as the basis density. The use of the sextic non-Gaussian measure density permits to obtain the rapidly converging series (2.26). Having (2.26), we can write and study the approximate RR. On the basis of (2.14) and RR, the free energy $F=-k_{\mathrm{B}} T \ln Z$ can be calculated.

\section{Discussion and conclusions}

The results obtained for 3D Ising model in an external field can be applied to the description of liquid-gas critical points of both a one-component fluid [2, 14] and a binary fluid mixture (see, for example, [15]). The CV method with a reference system (RS) is used for these systems. The expression for the partition function contains the even and the odd powers of the variable with the corresponding cumulants and is similar to (2.4), (2.5). Detailed investigation of the properties of $\mathrm{RS}$ cumulants in [2] makes it possible to transform the grand partition function into a functional form defined on the effective block lattice. The functional corresponds to the partition function of the Ising model in an external field. A new point in the problem of the liquid-gas critical point as compared to the case of the Ising model is the dependence of the critical temperature and of all coefficients on the density and chemical potential. The latter is equivalent to the insertion of a constant external field into the Ising model. To describe the effects connected with the asymmetry for the surface of coexistence of the phases, one should compute the partition function using the sextic measure density $[2,16]$.

In this paper, the 3D one-component spin system in a nonzero external field is investigated using the CV method in the asymmetric $\rho^{6}$ model approximation. An initial expression for the partition function of the system is constructed in the form of a functional with explicitly known coefficient functions (see (2.7)). The partition function is integrated over the layers of the CV phase space. The main feature is the integration of short-wave spin density oscillation modes, which is generally done without using perturbation theory. The short-wave modes are characterized by the presence of the RG symmetry and are described by the non-Gaussian measure 
density. The general RR (2.22) for the coefficients of the even and the odd powers of the variable in the two adjacent sextic measure densities are found. The new special functions appearing in the construction of the theory using the asymmetric $\rho^{6}$ model are considered. These functions entering the $\mathrm{RR}$ are represented in the form of a power series in small deviations of the basic arguments from their values at a fixed point. Representing the RR in the form of a nonasymptotic series relates to rejecting the traditional use of perturbation theory, which is based on the Gaussian measure density.

The expressions under investigation allow us to correctly trace the dependence of the results on a field within the framework of the CV method. As a consequence of the presence of an external field $h$ in the Hamiltonian (2.1), we obtain odd powers of the variable in the expressions (2.5) for the Jacobian $J(\rho)$ and (2.7) for the partition function $Z$. The cumulants $\mathcal{M}_{n}$ in the Jacobian (2.5) are combinations of the quantities $x=\tanh h^{\prime}$ and $y=1-\tanh ^{2} h^{\prime}$, where $h^{\prime}=\beta h$ is a generalized field. The initial coefficients $a_{l}^{\prime}(2.9)$ of the partition function are determined by these cumulants and are depended by $s_{0}$ on the microscopic parameters of the system (the effective interaction radius $b$ and the lattice constant $c$ ). The cumulants $\mathcal{M}_{1}, \mathcal{M}_{3}$, $\mathcal{M}_{5}$ as well as the coefficients $a_{1}^{\prime}, a_{3}^{\prime}, a_{5}^{\prime}$ are odd functions of an external field. The quantities $\mathcal{M}_{2}, \mathcal{M}_{4}, \mathcal{M}_{6}$ and $a_{0}^{\prime}, a_{2}^{\prime}, a_{4}^{\prime}, a_{6}^{\prime}$ are even functions of a field. The RR (2.22) make it possible to determine the elements of the block Hamiltonians from the initial data. As follows from the obtained expressions, the cumulants $\mathcal{M}_{1}, \mathcal{M}_{3}, \mathcal{M}_{5}$ and coefficients $a_{1}^{\prime}, a_{3}^{\prime}, a_{5}^{\prime}, \ldots, a_{1}^{(n)}, a_{3}^{(n)}, a_{5}^{(n)}$ vanish at $h=0$. Then the RR (2.22) for the asymmetric $\rho^{6}$ model turn into the RR for the $\rho^{6}$ model with even powers only [13]. In the case when $h \neq 0$, the measure density involves odd powers of the variable $\rho_{\mathbf{k}}$ in addition to the even powers. Although the CV method as well as Wilson's approach exploit the RG ideas, it is based on the use of non-Gaussian measure densities. This allows one to obtain a qualitatively new form of the RR between the coefficients of the block Hamiltonians. In the limiting case (corresponding to the Gaussian basis measure density; $h=0$ ) these $R R$ reduce to the Wilson $R R$ [11]. As was shown in [17], while this limiting case does not allow one to perform the calculation of the expression for the free energy of the system, it provides reliable results for the critical exponents of thermodynamic characteristics. The solutions of the RR under consideration can be used for calculating the thermodynamic functions of the system and for deriving the equation of state using the method proposed in [18].

\section{References}

1. Yukhnovskii I.R. Phase Transitions of the Second Order. Collective Variables Method. Singapore, World Scientific, 1987.

2. Yukhnovskii I.R., Idzyk I.M., Kolomiets V.O. // J. Stat. Phys., 1995, vol. 80, No. 1/2, p. 405-443.

3. Yukhnovskii I.R., Patsahan O.V. // J. Stat. Phys., 1995, vol. 81, No. 3/4, p. 647-672.

4. Vause C., Sak J. // Phys. Rev. A, 1980, vol. 21, No. 6, p. 2099-2114.

5. Nicoll J.F. // Phys. Rev. A, 1981, vol. 24, No. 4, p. 2203-2220. 
6. Pylyuk I.V. // Theor. Math. Phys., 1998, vol. 117, No. 3, p. 1459-1482.

7. Kozlovskii M.P., Pylyuk I.V., Dukhovii V.V. // Cond. Matt. Phys., 1997, No. 11, p. $17-49$.

8. Pylyuk I.V. // Low Temp. Phys., 1999, vol. 25, No. 11, p. 877-888.

9. Pylyuk I.V. // Low Temp. Phys., 1999, vol. 25, No. 12, p. 953-961.

10. Ma Shang-keng. Modern Theory of Critical Phenomena. Reading, Massachusetts, Benjamin, 1976.

11. Wilson K.G., Kogut J. // Phys. Rep. C, 1974, vol. 12, No. 2, p. 75-199.

12. Kozlovskii M.P., Pylyuk I.V., Usatenko Z.E. // Phys. Stat. Sol. (b), 1996, vol. 197, p. $465-477$.

13. Kozlovskii M.P. // Theor. Math. Phys., 1989, vol. 78, No. 3, p. 300-308.

14. Yukhnovskii I.R. // Physica A, 1990, vol. 168, No. 3, p. 999-1020.

15. Kozlovskii M.P., Patsahan O.V. // Cond. Matt. Phys., 2000, vol. 3, No. 3(23), p. 607631.

16. Yukhnovskii I.R. // Proceedings of the Steklov Institute of Mathematics, 1992, Iss. 2, p. 223-243.

17. Ilnytskyi Ya.M., Kozlovskii M.P., Yukhnovskii I.R. // Int. J. Mod. Phys. B, 1997, vol. 11, No. 8, p. 1009-1022.

18. Kozlovskii M.P., Pylyuk I.V., Dukhovii V.V. // J. Magn. Magn. Mater., 1997, vol. 169, p. $335-342$.

\title{
Тривимірна ізінгівська система в зовнішньому полі. Рекурентні співвідношення для асиметричної моделі $\rho^{6}$
}

\author{
І.В.Пилюк, М.П.Козловський \\ Інститут фізики конденсованих систем НАН України, \\ 79011 Львів, вул. Свєнціцького, 1
}

Отримано 26 липня 2000 р.

Тривимірна однокомпонентна спінова система в зовнішньому магнітному полі досліджується з допомогою методу колективних змінних. Інтегрування статистичної суми системи по шарах фазового простору здійснюється в наближенні густини міри, яка містить в собі парні та непарні степені змінної до шостого включно (асиметрична модель $\rho^{6}$ ). Одержано загальні рекурентні співвідношення між коефіцієнтами ефективних густин мір. Нові функції, що появляються в цих рекурентних співвідношеннях, подані у вигляді збіжних рядів.

Ключові слова: модель Ізінга, зовнішнє поле, колективні змінні, рекурентні співвідношення

PACS: 05.50. $+q, 75.10 . \mathrm{Hk}$ 